\title{
Editorial
}

\section{The Microbiology of Bacterial Lung Abscess: Time for a Reappraisal?}

\author{
Ioannis Kioumis $^{a}$ Demosthenes Bouros ${ }^{b}$ \\ Departments of Pneumonology, Medical Schools of a Aristotle University of Thessaloniki and \\ ${ }^{b}$ Democritus University of Thrace, Alexandroupolis, Greece
}

The microbial etiology of lung abscess is thought to be well documented and the organisms considered to be predominantly responsible consist of the (mostly anaerobic) normal flora in the mouth and, more specifically, gingival crevices [1]. A number of studies using sample collection techniques that avoid contamination with oral flora, in combination with efficient anaerobic culture methods, have shown that anaerobes participate in up to $90 \%$ of the lung abscesses [2]. Notably, these organisms are the only pathogens present in about half of the cases. Usually, lung abscesses contain multiple anaerobe species [3].

Monomicrobial abscesses are only occasionally identified and may be caused by bacteria such as Staphylococcus aureus, Pseudomonas aeruginosa, Klebsiella spp., Pasteurella multocida, Enterobacteriaceae, Haemophilus influenzae (types b and c), Actinomyces spp., Nocardia spp., Rhodococcus equi, Burkholderia pseudomallei, Streptococcus anginosus group, Streptococcus pneumoniae, Legionella, etc. Physicians should avoid misdiagnosing a tuberculous cavitary lesion as a lung abscess, as most signs and symptoms are nonspecific [4]. Anyhow, most of the publications containing data on the etiology of lung abscess are more than a quarter of a century old, with new information scarce and sketchy.

It is beyond any doubt that both the incidence and the mortality of lung abscess are constantly declining. In fact, while studies published in the mid-1980s steadily report-

\section{KARGER}

Fax +4161306 1234

E-Mail karger@karger.ch

www.karger.com (c) 2010 S. Karger AG, Basel

0025-7931/10/0802-0096\$26.00/0

Accessible online at:

www.karger.com/res ed an overall mortality around a dismal 15-20\% [5, 6], the most recent studies dealing with primary lung abscesses (including the one published here below) report a substantial reduction of the mortality which, at present, does not seem to exceed a very encouraging 1-5\% [7]. However, it must be emphasized that mortality is related not only to the underlining medical conditions, but also to certain predisposing factors, including the size and the topography of the lesion, as well as to the involvement of highly virulent pathogens (e.g. S. aureus, Klebsiella pneumoniae, $P$. aeruginosa) [8]. Therefore, a potential increment of the implication of any of these (or other) particularly virulent pathogens could have a dramatic impact on actual mortality.

New insight on the microbiology of lung abscess came in 2005 from the study by Wang et al. [9]. Quite surprisingly, the authors found that the bacteriology of this destructive infection (at least in Taiwan) has changed, as the contribution of the anaerobes was limited to $31 \%$ of the cases, while $K$. pneumoniae was the predominant bacterium in $33 \%$ of the patients with community-acquired lung abscess. Despite the fact that the number of the subjects was moderate (90), an advantage of the study was that microbiology specimens were obtained by the use of transthoracic aspiration. Of course, a direct consequence of these results (if they are confirmed and geographically generalized by similar prospective studies) would be a 
change of the selection of antibiotics for the treatment of lung abscess, which hereafter should include coverage of $K$. pneumoniae. Perhaps a key factor for the correct interpretation of these results is that $K$. pneumoniae strains in Taiwan appear to be more virulent than strains from other parts of the world [10].

The study of Takayanagi et al. [11] published in this issue of Respiration, brings some additional information about the changing etiology of lung abscess, although not in line with the previous study. The authors report the results of a retrospective analysis of data obtained from 122 of 205 Japanese patients with community-acquired lung abscess to which bacteriologic documentation was possible. Despite the diversity of the applied methodology of sampling and the high percentage $(47.8 \%)$ of prior antibiotic use, which undoubtedly compromise (at least to some extent) the accuracy of the results, the study reports an impressively low incidence of anaerobic bacteria, either as a sole or participating causative agent (26.2\%). Streptococcus species were isolated in 85 of 107 patients, while K. pneumoniae (contrary to the study of Wang et al. [9]) was recovered in only 10 patients $(8.2 \%)$ despite the fact that $16.6 \%$ were alcohol drinkers and $22.9 \%$ were diabetics.

Another striking finding might be the recovery of $\mathrm{Ge}$ mella species in $9.8 \%$ of the aerobic isolates. Gemella species are rarely reported as the causative agents of lung abscess. A possible explanation for this disparity could be the inability of some laboratories to discriminate Gemella spp. from other viridans of Streptococci. On the other hand, it is known that both Vitec (GPI) and API (20 STREP) systems not rarely identify incorrectly Granulicatella adiacens and Abiotrophia defectiva as Gemella species [12]. Formerly considered as 'nutritionally defective' or 'satelliting' streptococci, these are more difficult to eradicate with antimicrobials; therefore, they could resist prior antibiotic administration, as was the case with nearly half of the study's patients. It is worthy to mention that in a European study performed by García-Lechuz et al. [13], Gemella was recognized to cause only one case of lung abscess out of 128 patients with a variety of extraabdominal infections due to this microbe.

So, where are we? Are we to believe that this diversity of lung abscess bacteriology is a geographic phenomenon or that it is just an artifact due to methodological problems? Could it also be possible that these observed differences reflect the divergent polities on selection and use of antibiotics that are implemented in different countries? These are questions that have to be answered as soon as possible, as they imply possible changes in the therapeutic approach. We need fresh, prospective and well-designed studies, employing reliable methodology for specimen sampling, to look thoroughly at the etiology of lung abscesses for the second decade of the 21st century. The 'old' is not always 'good'.

\section{References}

1 Bartlett JG: Anaerobic bacterial infections of the lung and pleural space. Clin Infect Dis 1993;16(suppl 4):S248-S255.

2 Brook I, Finegold SM: Bacteriology and therapy of lung abscess in children. J Pediatr 1979;94:10.

3 Bartlett JG, Gorbach SL, Tally FP, et al: Bacteriology and treatment of primary lung abscess. Am Rev Respir Dis 1974;109:510-518.

-4 Israel RH, Poe RH, Greenblatt DW, et al: Differentiation of tuberculous from nontuberculous cavitary lung disease. Respiration 1985;47:151-157.

5 Pohlson EC, McNamara JJ, Char C, et al: Lung abscess: a changing pattern of disease. Am J Surg 1985;150:97-101.
6 Hagan JL, Hardy JD: Lung abscess revisited: a survey of 184 cases. Ann Surg 1983;197: 755-762.

7 Moreira JDS, Camargo JDJP, Felicetti JC, et al: Lung abscess: analysis of 252 consecutive cases diagnosed between 1968 and 2004. J Braz Pneumol 2006;32:136-143.

8 Hirshberg B, Sklair-Levi M, Nir-Paz R, et al: Factors predicting mortality of patients with lung abscess. Chest 1999;115:746-750.

-9 Wang JL, Chen KY, Fang CT, et al: Changing bacteriology of adult community-acquired abscess in Taiwan: Klebsiella pneumoniae versus anaerobes. Clin Infect Dis 2005;40: 915-922.
$10 \mathrm{Yu}$ VL, Chang F-Y: The role of Klebsiella pneumoniae in lung abscesses: a geographic phenomenon (for now!). Clin Infect Dis 1006;42:152-153.

11 Takayanagi N, Kagiyama N, Ishiguro T, et al: Etiology and outcome of community-acquired lung abscess. Respiration 2010;80: 98-105.

12 Woo PCY, Lau SKP, Fung AMY et al: Gemella bacteremia characterized by $16 \mathrm{~S}$ ribosomal RNA gene sequencing. J Clin Pathol 2003; 56:690-693.

13 García-Lechuz JM, Cuevas-Lobato O, Hernángomez $\mathrm{S}$, et al: Extra-abdominal infections due to Gemella species. Int J Infect Dis 2002;6:78-82. 